\title{
The divergence history of European blue mussel species reconstructed from Approximate Bayesian Computation: the effects of sequencing techniques and sampling strategies
}

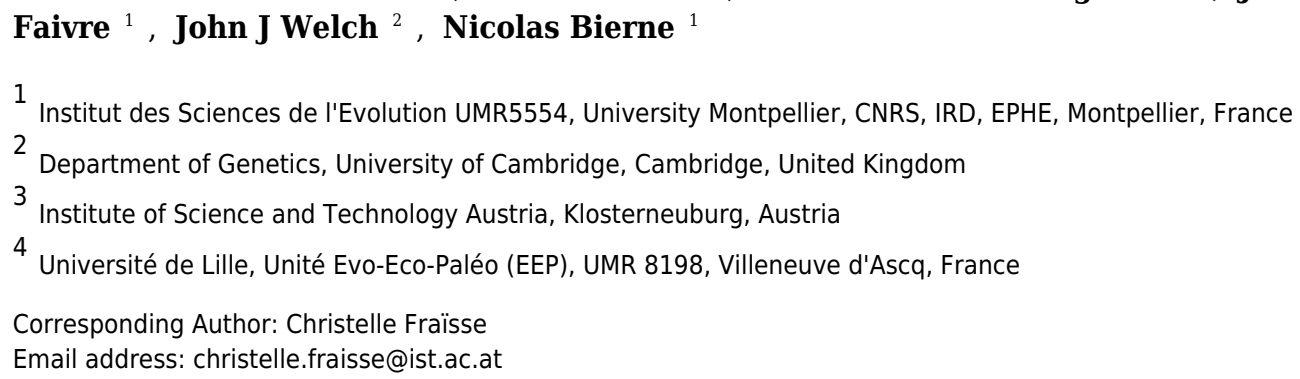

Genome-scale diversity data are increasingly available in a variety of biological systems, and can be used to reconstruct the past evolutionary history of species divergence. However, extracting the full demographic information from these data is not trivial, and requires inferential methods that account for the diversity of coalescent histories throughout the genome. Here, we evaluate the potential and limitations of one such approach. We reexamine a well-known system of mussel sister species, using the joint site frequency spectrum (jSFS) of synonymous mutations computed either from exome capture or RNA-seq, in an Approximate Bayesian Computation (ABC) framework. We first assess the best sampling strategy (number of: individuals, loci, and bins in the jSFS), and show that model selection is robust to variation in the number of individuals and loci. In contrast, different binning choices when summarizing the jSFS, strongly affect the results: including classes of low and high frequency shared polymorphisms can more effectively reveal recent migration events. We then take advantage of the flexibility of $A B C$ to compare more realistic models of speciation, including variation in migration rates through time (i.e. periodic connectivity) and across genes (i.e. genome-wide heterogeneity in migration rates). We show that these models were consistently selected as the most probable, suggesting that mussels have experienced a complex history of gene flow during divergence and that the species boundary is semi-permeable. Our work provides a comprehensive evaluation of $A B C$ demographic inference in mussels based on the coding jSFS, and supplies guidelines for employing different sequencing techniques and sampling strategies. We emphasize, perhaps surprisingly, that inferences are less limited by the volume of data, than by the way in which they are analyzed. 
1 The divergence history of European blue mussel species

2 reconstructed from Approximate Bayesian Computation: the

3 effects of sequencing techniques and sampling strategies

5 Christelle Fraïsse ${ }^{1,2,3, *}$, Camille Roux ${ }^{4}$, Pierre-Alexandre Gagnaire ${ }^{1}$, Jonathan Romiguier ${ }^{1}$,

6 Nicolas Faivre $^{1}$, John J. Welch ${ }^{2}$, Nicolas Bierne ${ }^{1}$

$7{ }^{1}$ Université Montpellier, Institut des Sciences de l'Évolution, UMR 5554, Montpellier Cedex 05,

8 France.

92 Department of Genetics, University of Cambridge, Downing Street, Cambridge, UK.

103 Institute of Science and Technology Austria, Am Campus 1, 3400 Klosterneuburg, Austria.

114 Université de Lille, Unité Evo-Eco-Paléo (EEP), UMR 8198, Villeneuve d'Ascq Cedex, F12 59655, France.

13 * Corresponding author: christelle.fraisse@ist.ac.at

15 Short title: Evaluating spectrum-based $\mathrm{ABC}$ inference in mussels 
16 Abstract

17 Genome-scale diversity data are increasingly available in a variety of biological systems, and can

18 be used to reconstruct the past evolutionary history of species divergence. However, extracting

19 the full demographic information from these data is not trivial, and requires inferential methods

20 that account for the diversity of coalescent histories throughout the genome. Here, we evaluate

21 the potential and limitations of one such approach. We reexamine a well-known system of mussel

22 sister species, using the joint site frequency spectrum (jSFS) of synonymous mutations computed

23 either from exome capture or RNA-seq, in an Approximate Bayesian Computation (ABC)

24 framework. We first assess the best sampling strategy (number of: individuals, loci, and bins in

25 the jSFS), and show that model selection is robust to variation in the number of individuals and

26 loci. In contrast, different binning choices when summarizing the jSFS, strongly affect the results:

27 including classes of low and high frequency shared polymorphisms can more effectively reveal

28 recent migration events. We then take advantage of the flexibility of $\mathrm{ABC}$ to compare more

29 realistic models of speciation, including variation in migration rates through time (i.e. periodic

30 connectivity) and across genes (i.e. genome-wide heterogeneity in migration rates). We show that

31 these models were consistently selected as the most probable, suggesting that mussels have

32 experienced a complex history of gene flow during divergence and that the species boundary is

33 semi-permeable. Our work provides a comprehensive evaluation of $\mathrm{ABC}$ demographic inference

34 in mussels based on the coding jSFS, and supplies guidelines for employing different sequencing

35 techniques and sampling strategies. We emphasize, perhaps surprisingly, that inferences are less

36 limited by the volume of data, than by the way in which they are analyzed. 


\section{Introduction}

41 The biodiversity we inherited from the Quaternary was shaped by the process of species

42 formation (Hewitt 2000). A long-standing question concerns the timing and rate of gene exchange

43 that occurred while populations diverged, during the incipient stages of speciation. Model-based

44 inferences from genetic data have been used to investigate the history of gene flow (Beaumont $e t$

45 al. 2010). Special attention has been paid to the distinction between recent divergence in a strict

46 isolation model, and older divergence with continuous migration (Nielsen \& Wakeley 2001),

47 although of course, more complex scenarios are also possible (Marino et al. 2013, Sousa \& Hey

48 2013).

49 With next-generation sequencing technologies, thousands of SNPs throughout the genome can be 50 used to infer the demographic histories of non-model species pairs (Sousa \& Hey 2013). One way

51 of summarizing the information in these data is the unfolded joint site frequency spectrum (jSFS),

52 i.e. the number of copies of derived alleles found in each of the two sampled species. A recent

53 and fast maximum-likelihood method based on the jSFS (Gutenkunst et al. 2009) has proven

54 useful for distinguishing continuous migration from strict isolation (e.g. in ragworts, Chapman et

55 al. 2013, and beach mice, Domingues et al. 2012). The method can also evaluate more complex

56 scenarios (e.g., in sea bass, Tine et al. 2014; poplars, Christe et al. 2017; and whitefish, Rougeux

57 et al. 2017), but it struggles to explore the parameter space in these cases. In addition, the method

58 is not well suited for transcriptome data as model comparison by log-likelihood ratio tests

59 assumes independence of SNPs. As a consequence simulations need to be conducted to evaluate

60 competing models, and the computational speed advantage is lost.

61 As an alternative, Approximate Bayesian Computation (ABC) is a method based on simulations

62 that avoids the need to explicitly compute the likelihood (Beaumont et al. 2002). As such,

63 histories of speciation characterized both by periods of strict isolation and periods of gene 
64 exchange can easily be investigated, e.g. the scenarios of ancient migration and secondary

65 contact. These scenarios can be extended by including two cycles of "isolation / gene exchange",

66 following climatic changes in the Pleistocene (Figure 1). Methods have also been developed to

67 include genome-wide heterogeneity in migration rates (Sousa et al. 2013; Roux et al. 2013). This

68 is consistent with the "genic view" of speciation (Wu 2001), whereby barriers to gene flow are

69 often semi-permeable, varying in strength across the genome due to linked selection and

70 recombination (Barton \& Bengtsson 1986). A major challenge in ABC, as compared to explicit

71 likelihood methods, is the selection of summary statistics, which involves a trade-off between

72 loss of information and reduction of dimensionality. Several methods have been suggested to

73 select the most appropriate statistics for a given dataset and a set of models (e.g., Wegmann et al.

74 2009; Nunes \& Balding 2010; Aeschbacher et al. 2012); but most of these summarize the SFS

75 (but see, e.g. Boitard et al. 2016) leading to a loss of information. Recently, several ABC studies

76 have used the SFS directly to reconstruct the history of single populations (Boitard et al. 2016),

77 or multiple populations (Xue \& Hickerson 2015; Smith et al. 2017). However the number of

78 statistics, i.e. the number of classes in the spectrum, increases quadratically with the number of

79 haploid genomes sampled in the case of a two-dimensional SFS, and even faster if more than two

80 populations are considered. For this reason, recent studies have tested different ways of binning

81 the SFS to circumvent the curse of dimensionality (e.g., Smith et al. 2017). While accumulating

82 new data and developing new methods, studies that evaluate the impact of the sampling strategy

83 and the inferential method (e.g. Li \& Jakobsson 2012; Robinson et al. 2014; Shafer et al. 2015;

84 Cabrera \& Palsbøll 2017; Smith et al. 2017) to reconstruct species divergence history will be

85 increasingly valuable.

86 Mytilus edulis (Linnaeus, 1758) and Mytilus galloprovincialis (Lamarck, 1819) are two closely-

87 related species that currently hybridize where their ranges overlap along the Atlantic French

88 coasts (Bierne et al. 2003a) and the British Isles (Skibinski et al. 1983). Their interspecific barrier 
89 to gene flow is semi-permeable, and it has been shown to involve multiple isolating mechanisms,

90 both pre-zygotic (e.g. assortative fertilization and habitat choice, Bierne et al. 2002, 2003b), and

91 post-zygotic (hybrid fitness depression, Simon et al. 2017). Other evidence of ongoing gene flow

92 between $M$. edulis and M. galloprovincialis comes from footprints of local introgression of

93 edulis-derived alleles into a population of $M$. galloprovincialis enclosed within the Atlantic

94 hybrid zone (Fraïsse et al. 2014). Another study (Fraïsse et al. 2016) revealed that the Atlantic

95 population of $M$. galloprovincialis was more introgressed than the Mediterranean population on

96 average. At some specific loci, however, the Mediterranean population was found to be fixed for

97 edulis alleles while the Atlantic population was not introgressed at all, suggesting that an ancient

98 contact between M. edulis and Mediterranean M. galloprovincialis occurred during glacial

99 periods. Finally, direct model comparisons have been conducted with the IMa method of Hey \&

100 Nielsen (2007), and with an ABC framework, and shown that M. edulis and M. galloprovincialis

101 have experienced a complex history of divergence punctuated by periods of gene flow in Europe

102 (IMa: Boon et al. 2009, ABC: Roux et al. 2014, 2016).

103 Here, we analysed coding sequence datasets from this well-known pair of sister species in

104 Europe, and systematically reconstructed its speciation history by ABC, using different sampling

105 strategies. By different sampling strategies, we mean that we varied (i) the number of individuals

106 sampled (2, 4, or 8), (ii) the number of SNPs (which were obtained by different sequencing

107 techniques, "exome capture" vs. "rna-seq") and (iii) the number of bins in the jSFS (binning the

108 spectrum into 4,7 or 23 classes). We then evaluated the influence of these choices on model

109 selection, using eleven distinct scenarios of speciation. Our results show that the influence on

110 inferences of the number of individuals and loci sampled is surprisingly limited, while the

111 different ways of binning the jSFS strongly affect the results. This suggests that demographic

112 reconstructions are nowadays more limited by the way data are analyzed than by their volume.

113 Moreover, we find that an history of periodic connectivity, with both ancient and contemporary 
114 introgression, and a semi-permeable barrier to gene flow, best fit these data, arguing for the

115 development of flexible inference methods to better describe complex divergence histories

116 (Simon \& Duranton 2018).

119 Materials and Methods

\section{Sampling, sequencing, mapping and calling}

121 Two datasets were analysed for demographic inferences. They both comprise measures of 122 molecular polymorphism and divergence in coding sequences, obtained for the pair M. edulis and 123 M. galloprovincialis. Although the adopted sequencing techniques were different among datasets 124 ("exome capture" vs. "rna-seq"), the surveyed populations were similar. The usage of coding 125 sequences to infer divergence histories of closely-related species (e.g., see Roux et al. 2013, 126 2014, 2016; McCoy et al. 2014; Li et al. 2017; Qi et al. 2017) is justified for several reasons: (i) 127 synonymous mutations are less affected by direct selection than other categories of mutation, and 128 selection affects chromosomal regions larger than genes themselves, including non-coding 129 regions (e.g., regulatory elements, Andolfatto 2005); (ii) we implicitly modelled the effects of 130 selection against migrant genes by including heterogeneous effective migration rates across the 131 genome.

\section{Data set 1: "exome capture"}

134 We used the dataset already published in Fraïsse et al. (2016) and available on 135 http://www.scbi.uma.es/mytilus/index.php. Briefly, a set of 890 EST contigs was used as a 136 reference for a pre-capture multiplex DNA enrichment in samples of eight individuals from two 137 geographical populations in each species (M. edulis: North Sea and Bay of Biscay; $M$. 
138 galloprovincialis: Brittany and Mediterranean Sea, Table 1). In addition, we used a sample of

139 four individuals of $M$. trossulus to serve as an outgroup (Table 1). Each DNA library was

140 sequenced twice to increase the per-base coverage (Miseq or GA2X followed by HiSeq2000).

141 After trimming and quality-filtering, reads of each individual were aligned against the same EST

142 reference sequences using the BWA program (bwa-mem, Li \& Durbin 2009). Because of the

143 relative divergence between the two species $(\sim 2 \%$, Table 2$)$, we adjusted the default parameter of

144 BWA to allow less stringent mapping (minimum seed length $k=10$ [default: 19], clipping penalty

$145 L=3[5]$, mismatch penalty $B=2$ [4], and gap open penalty $O=3$ [6]). Full methods are described in

146 Fraïsse et al. (2016).

147 We used a maximum-likelihood method, implemented in the program read2snps (Tsagkogeorga

148 et al. 2012; Gayral et al. 2013), to call genotypes directly from read numbers at each position.

149 The method computes the probability of each possible genotype after estimating the sequencing

150 error rate. To limit bias in the site frequency estimation (Han et al. 2013), a minimum of 10X

151 coverage, i.e. ten reads per position and per individual, was required to call a genotype. Only

152 genotypes supported at 95\% were retained; otherwise missing data was applied. Moreover,

153 paralogous positions were filtered-out using a likelihood ratio test based on explicit modelling of

154 paralogy.

155

156 Data set 2: "rna-seq"

157 The dataset 2 is made of sequenced transcriptomes (RNA-seq) previously generated in a wide

158 meta-analysis study comparing levels of polymorphism across 76 animal species (Romiguier et

159 al. 2014). It includes the transcriptomes of four individuals sampled in the same populations as

160 described above and one individual of M. trossulus (Table 1). Briefly, for each individual, cDNA

161 libraries were prepared with total RNA extracted from whole body and sequenced on HiSeq2000.

162 Illumina reads (100 bp, paired-end) were mapped with the BWA program on de novo 
163 transcriptomes, independently assembled for each species with a combination of the programs

164 Abyss and Cap3, following the strategy B and D in Cahais et al. (2012). Contigs with a per-

165 individual average coverage below $\times 2.5$ were discarded. Genotype calling was performed as

166 described in the first data set using identical filters. Open reading frames were predicted with the

167 Trinity package and sequences carrying no ORF longer than 200 bp were discarded. Full methods

168 are described in Romiguier et al. (2014).

\section{$\underline{\text { Data analysis }}$}

171 We restricted our analysis to loci assembled in all individuals, and longer than $300 \mathrm{bp}$ after

172 filtering positions containing missing data, or more than two segregating alleles when the three 173 species were aligned. Only synonymous positions were used. The total number of loci and SNPs 174 retained in each dataset is given in Table 2.

175

176

177

\section{Site Frequency Spectrum}

We first computed the jSFS for each dataset (Figure 2 and Figure S1). Each derived allele, oriented by treating the outgroup sequence as a fixed ancestral allele, was assigned to one cell of the jSFS, depending on its frequency in each of the two populations. From the full jSFS, different classes of polymorphism were extracted and used as summary statistics (Tellier et al. 2011). Specifically, we used the four Wakeley-Hey classes (jsf $s=4$ in Figure 2): fixed differences, Sf; private polymorphisms for each species $\mathrm{Sx}_{1}$ and $\mathrm{Sx}_{2}$; and shared polymorphisms, Ss (Wakeley \& Hey 1997). We also considered a summary in which the Sf and Sx classes were split depending on whether the derived allele was fixed or absent in the other species ( $j s f s=7$ in Figure 2; Ramos-

Onsins et al. 2004). The third decomposition of the jSFS contains twenty-three classes of polymorphisms because singletons and doubletons in each population were included as new classes $\left(j s f_{s}=23\right.$ in Figure 2). This corresponds to the full jSFS with $n=2$ diploid individuals. 
190 Estimators of polymorphism and divergence

191 We then computed a set of genetic statistics across loci to make use of the coalescent information 192 contained within each sequence, instead of considering each SNP separately. Following previous 193 studies (e.g., Fagundes et al. 2007; Ross-Ibarra et al. 2008; Roux et al. 2011), we used the 194 following statistics: (1) nucleotide diversity, $\pi_{1}$ and $\pi_{2}$ (Tajima 1983); (2) Watterson's $\theta_{\mathrm{w} 1}$ and $\theta_{\mathrm{w} 2}$ 195 (Watterson 1975); (3) total and net interspecific divergence, div and netdiv; (4) between-species 196 differentiation, FST, computed as $1-\pi_{\mathrm{S}} / \pi_{\mathrm{T}}$, where $\pi_{\mathrm{S}}$ is the average pairwise nucleotide diversity 197 within species and $\pi_{\mathrm{T}}$ is the total pairwise nucleotide diversity of the pooled sample across 198 species. We also included the four Wakeley-Hey's classes as explained above ( $\mathrm{Sf}, \mathrm{Sx}_{1}, \mathrm{Sx}_{2}$ and 199 Ss). Finally, we assessed departure from mutation/drift equilibrium using Tajima's $\mathrm{D}_{1}$ and $\mathrm{D}_{2}$ 200 (Tajima 1989a,b). The average and standard deviation across the loci of these statistics were 201 calculated with the program MScalc (available from http://www.abcgwh.sitew.ch/, see Roux et al. 202 2011), and their values are given in Table 2 ("mscalc").

\section{Inferences by Approximate Bayesian Computation}

\section{Scenarios of speciation}

206 Six distinct scenarios of speciation were considered (Figure 1). Each scenario modeled an

207 instantaneous division (occurring $\mathrm{T}_{\text {split }}$ generations ago) of the ancestral population of effective 208 size $\mathrm{N}_{\mathrm{A}}$ into two populations of constant sizes $\mathrm{N}_{1}$ and $\mathrm{N}_{2}$. The Strict Isolation scenario (SI) 209 assumed that divergence occurred without gene exchange between the two populations. The other 210 models differed by their temporal pattern of migration, which occurred at a rate $\mathrm{M}_{12}$ from 211 population 1 to population 2, and a rate $\mathrm{M}_{21}$ in the opposite direction. Ancient Migration (AM) 
212 and Periodic Ancient Migration (PAM) scenarios both assumed that migration was restricted to

213 the early period of divergence. In the AM scenario, the two populations experienced a single

214 period of strict isolation $\left(\mathrm{T}_{\text {iso }}\right)$ while in the PAM scenario, migration was stopped twice with an

215 intermediate period of isolation of $\mathrm{T}_{\text {iso }} / 2$ generations. In the Isolation Migration (IM), Secondary

216 Contact (SC) and Periodic Secondary Contact (PSC) scenarios, gene exchange was currently

217 ongoing between the two populations. In the SC and PSC scenarios, the two populations first

218 evolved in strict isolation and then experienced a period of gene exchange $\left(\mathrm{T}_{\mathrm{sc}}\right)$. In the $\mathrm{SC}$

219 scenario, there was a single period of recent migration whereas in the PSC scenario a period of

220 ancient migration also occurred after $1-\mathrm{T}_{\mathrm{sc}} / 2$ generations of strict isolation. The last scenario is

221 the standard isolation with migration (IM) scenario in which migration occurred continuously

222 over time since the two species started to diverge. For models including migration (IM, AM,

223 PAM, SC and PSC), we compared two alternative models in which the effective migration rate

224 was either homogeneous ("homo") or heterogeneous ("hetero") among loci (Roux et al. 2013,

225 2014, 2016). These models aim to account for the effects of a semi-permeable barrier to gene

226 flow: a reduced effective migration rate is predicted in chromosomal regions linked to

227 incompatible genes; while free introgression is predicted in loosely linked regions.

228

229

Coalescent simulations

230 For each of the five sampling strategies, we performed one million multilocus simulations under

231 the eleven scenarios of speciation using the coalescent simulator Msnsam (Hudson 2002; Ross-

232 Ibarra et al. 2008). Simulations fitted to the characteristics of each data set ("exome capture" data

233 with $n=2,4,8$ individuals and "rna-seq" data with $n=2,4$ individuals). We assumed free

234 recombination between contigs, and we fixed the intra-contig population recombination rate to be

235 equal to the population mutation rate. Previous studies have shown that methods which take intra-

236 locus recombination into account remain valid when rates of recombination are low (Becquet \& 
237 Przeworski 2007). Moreover, our method does not rely on haplotypic data, and so not estimating

238 exact rates of recombination should not affect our results. To account for errors in identifying the 239 ancestral allele in the unfolded jSFS, we explicitly modeled a misorientation rate in our

240 coalescent simulations. We assumed that a proportion $e$ of SNPs, which was a parameter to be

241 inferred, were misoriented and changed $e_{\mathrm{i}}$, their frequency in population i, into 1- $e_{i}$.

242 Prior distributions for $\theta_{A} / \theta_{\text {ref }}, \theta_{1} / \theta_{\text {ref }}$ and $\theta_{2} / \theta_{\text {ref }}$ were uniform on the interval $0-20$ with

$243 \theta_{r e f}=4 * N_{r e f}^{*} \mu$. The effective size of the reference population, $N_{r e f}$, used in coalescent simulations

244 was arbitrarily fixed to 100,000 . The mutation rate, $\mu$, was set to $2.763 * 10^{-8}$ per bp per generation

245 (Roux et al. 2014). The $T_{\text {split }} / 4 N_{\text {ref }}$ ratio was sampled from the interval $0-25$ generations, 246 conditioning the parameters $T_{i s o}$ and $T_{s c}$ to be uniformly chosen within the $0-T_{\text {split }}$ interval. For the

247 scenarios including migration, we used the scaled effective migration rates $M=4 N m$, where $m$ is

248 the fraction of the population made up of effective migrants from the other population at each

249 generation. In the homogeneous model, a single effective migration rate, shared by all loci but

250 differing in each direction of introgression, was sampled from an uniform distribution in the

251 interval 0-40. For the heterogeneous model, we assumed two categories of loci occurring in

252 proportion $p$ and (1-p). The parameter $p$ was sampled from an uniform distribution in the interval

$2530-1$. The first category of loci are neutral genes introgressing at a migration rate sampled from an

254 uniform distribution in the interval $0-40$. The second category comprises loci affected by the

255 barrier to gene flow, so that their effective migration rate was reduced by a gene flow factor, $g f f$,

256 compared to neutral loci (Barton \& Bengtsson 1986). The gff was sampled from a Beta

257 distribution with two shape parameters (alpha chosen in the interval 0.001-10 and beta chosen in 258 the interval 0.001-5). Prior distributions were computed using a modified version of the program

259 Priorgen (Ross-Ibarra et al. 2008) as described in Roux et al. (2013). 
262 Model choice

263 To choose the best supported model, we followed the methods previously described in Roux et

264 al. $(2013,2014)$. Briefly, posterior probabilities for each of the eleven speciation scenario were

265 estimated with a neural network using the R package abc (Csilléry et al. 2012). It implements a

266 nonlinear multivariate regression by considering the model itself as an additional parameter to be

267 inferred. The $0.01 \%$ replicate simulations nearest to the observed values of the summary statistics

268 were selected. Moreover, to evaluate the relative probability of the heterogeneous model of 269 migration rates across loci, we compared the alternative models ("homo" vs. "hetero") within

270 each scenario including gene exchange. We approximated Bayes Factors, $B F$, as the posterior

271 probability of the best supported model divided by that of the model with the highest posterior

272 probability from the remaining candidates. The posterior probability of each model calculated

273 among the eleven models of speciation are detailed in Table S1; and those of the best model

274 between heterogeneous and homogeneous migration rates are given in Table S2.

276 Model checking

277 We checked the ability of our ABC framework to correctly recover the true model by a "leave-

278 one-out cross validation" from our simulations. We randomly extracted 100 simulated datasets

279 from the million simulations performed for each model. For each of the 100 of datasets simulated

280 under a given model (i.e., pseudo-observed datasets), we applied the model choice procedure

281 described above to compute the posterior probability of all competitive models. The accuracy rate

282 for model $M$ was calculated as the proportion, among pseudo-observed data inferred to

283 correspond to model $M$, of those actually generated under model $M$. The ambiguity rate was

284 computed as the proportion of pseudo-observed data generated under model $M$ whose best model

285 was not strongly supported, i.e. its posterior probability was below an arbitrary threshold $\left(\mathrm{P}_{\min }\right)$

286 set to be one third above the expected value given the total number of models compared (1/11 for 
287 eleven models, $1 / 6$ for six models or $1 / 2$ for two models). The accuracy and ambiguity rates for 288 the "exome capture" data with $n=2$ individuals are provided in Table S3.

$291 \underline{\text { Results }}$

292 Patterns of polymorphism

293 We obtained polymorphism data for two mussels species, M. edulis and M. galloprovincialis, and 294 one outgroup (M. trossulus) in samples of increasing size ( $n=2, n=4$ and $n=8)$ and for the two 295 datasets ("exome capture" vs. "rna-seq"). The jSFS of each data set is shown in Figure S1, while 296 Table 2 gives summary statistics of genetic polymorphism.

297 The data produced by the two sequencing techniques differed in the total number of SNPs 298

$\left(n_{\text {snp }}=3,993(n=2) ; \mathrm{n}_{\text {snp }}=5092(n=4) ; n_{\text {snp }}=5000(n=8)\right.$ in the "exome capture" data; $n_{\text {snp }}=17,275$ $299(n=2) ; n_{\text {snp }}=17,902(n=4)$ in the "rna-seq" data). The substantially lower number of SNPs in the 300 "exome capture" data reflects the lower number of loci retained for the analysis due to a reduced 301 and more heterogeneous sequencing depth compared to the "rna-seq" data (while applying 302 identical coverage filters to call SNPs). However, the jSFS calculated from the two techniques 303 had a similar proportion of sites in the different classes (Pearson's correlation between jSFS: $304 \mathrm{R}^{2}=0.97, \mathrm{p}<0.0001$ for $n=2$ individuals; and $\mathrm{R}^{2}=0.99, \mathrm{p}<0.0001$ for $n=4$ individuals). Globally, 305 the mean level of genetic differentiation was quite low (FST 10\% in the "exome capture" data; 306 FST $\sim 18 \%$ in the "rna-seq" data, see Table 2), but highly variable across loci (standard deviation 307 was $\sim 16 \%$ and $\sim 24 \%$, respectively). A low proportion of sites $(<5 \%$ in the "exome capture" data 308 and $<10 \%$ in the "rna-seq" data) were fixed differences, while two to three times more SNPs 309 were polymorphic and shared between the two species (see Figure S1). The level of intraspecific 310 nucleotide diversity was elevated $\left(\pi_{\text {edu }}=\pi_{\mathrm{gal}}=0.016\right.$ in the "exome capture" data; $\pi_{\text {edu }}=0.038$ and 
$311 \pi_{\mathrm{gal}}=0.036$ in the "rna-seq" data for $n=2$, Table 2) and not significantly different between the two

312 species (non-significant Wilcoxon signed-rank test). Polymorphic sites were mainly private to

313 each species ( $\sim 80 \%$ of the sites), and mainly corresponded to low frequency classes. Moreover,

314 the jSFS was remarkably symmetric suggesting limited differences in population size and/or

315 migration rates between the two species. These patterns were consistent across sample sizes, but

316 there were some differences comparing the two techniques. Specifically, the "exome capture"

317 data set showed significantly lower level of divergence (Wilcoxon signed-rank test, $\mathrm{p}<0.0001$

318 between netdiv ${ }_{\text {capture }}=0.004$ and netdiv ${ }_{\text {rna-seq }}=0.02$ for $n=2$ individuals, Table 2 ) and average number

319 of fixed differences between species per locus (Wilcoxon signed-rank test, $\mathrm{p}<0.0001$ between

$320 \mathrm{Sf}_{\text {capture }}=0.322$ and $\mathrm{Sf}_{\text {rna-seq }}=0.810$ for $n=2$ individuals, Table 2 ). These discrepancies were most

321 likely due to the use of a single reference in the "exome capture" data resulting in the problematic

322 mapping of highly divergent alleles from the two species.

\section{Effects of the number of individuals and SNPs on model selection}

325 We carried out model selection between the various scenarios of speciation shown in Figure 1, 326 and asked whether the number of individuals and number of SNPs had an effect. Results are

327 shown in Table 3a, which reports the posterior probability of the best supported scenario for each

328 sampling strategy; and in Table $3 \mathrm{~b}$ which compares the posterior probability of homogeneous vs.

329 heterogeneous migration for the best supported scenario (see also Table S1 and S2 for full 330 details).

331 Firstly, we compared the "exome capture" and the "rna-seq" data which differ in the number of

332 SNPs sampled (3,993 SNPs and 17,275 SNPs, respectively); and we found that the best

333 supported scenario was the same for both data sets (Table 3a). For example, when considering

334 twenty-three classes in the jSFS $(j s f s=23)$, the best supported scenario always involved recent and

335 genome-wide heterogeneous migration between the two species. The heterogeneous periodic 
336 secondary contact was the most supported scenario; and the next best model described a very

337 similar history of secondary contact with a single period of gene exchange:

$338 B F=\mathrm{P}_{\mathrm{PSC} . \text { hetero }} / \mathrm{P}_{\text {SC.hetero }}=1.21$ with the "exome capture" data, $B F=\mathrm{P}_{\mathrm{PSC} . \text { hetero }} / \mathrm{P}_{\mathrm{SC} . \text { hetero }}=1.07$ with the

339 "rna-seq" data, in the case of $n=2$ individuals. With $n=4$ individuals, for which the next best

340 model also included gene flow, those numbers were $\mathrm{P}_{\text {PSC.hetero }} / \mathrm{P}_{\text {IM.hetero }}=1.09$ and

$341 \mathrm{P}_{\mathrm{PSC} . \text { hetero }} / \mathrm{P}_{\mathrm{IM} . h e t e r o}=1.37$. The same patterns were found when using different subsets of the jSFS

$342(j s f s=4$ and $j s f s=8$, Table 3a), except that the best supported scenario was then the periodic ancient

343 migration. Regarding genome-wide heterogeneity of migration rates (Table 3b), the "rna-seq"

344 data gave more support to the heterogeneous model (e.g., $B F=\mathrm{P}_{\text {AM.hetero }} / \mathrm{P}_{\text {AM.homo }}=1.25$ with $n=2$ and

$345 j s f=4)$ compared to the "exome capture" data $\left(B F=\mathrm{P}_{\mathrm{AM} . h o m o} / \mathrm{P}_{\mathrm{AM} . h e t e r o}=1.26\right)$. This is consistent with

346 the higher heterogeneity of the jSFS in the "rna-seq" data, involving a higher proportion of fixed

347 differences and shared polymorphic sites (Table 2 and Figure S1).

348 Secondly, we evaluated the effect of the number of individuals sampled. As with the number of

349 SNPs, it is clear that sample size had little effect. The best supported scenario remained 350 consistent across the different sampling size $(n=2,4$ or 8 individuals; Table $3 \mathrm{a})$. For example,

351 when considering twenty-three classes in the jSFS ( $j s f=23)$, the heterogeneous periodic

352 secondary contact scenario was the best supported model whatever the sampling size in both 353 datasets.

\section{Effects on model selection of the number of classes in the jSFS}

356 We next investigated the effects of binning the jSFS: (i) on model choice in the mussel datasets

357 (Figure 2 and Table 3), and (ii) on the ability of the method to discriminate between different

358 speciation scenarios based on simulated datasets (Table S3). Given the limited effects of the

359 sampling strategy, the $\mathrm{ABC}$ performance results are presented for a simulated dataset resembling

360 the "exome capture" data with $n=2$ individuals only. This allowed us consider the full jSFS when 
361 using twenty-three classes of polymorphism.

362

363

364 The scenarios with recent migration (SC, PSC and IM) all strongly supported heterogeneity of

365 migration rates; and this support tended to increase with the number of polymorphic classes 366

\section{Heterogeneity of migration rates} considered (Table S2). For example in the "exome capture" data with $n=2$ individuals, the relative probability of the heterogeneous model in the periodic secondary scenario was $\mathrm{P}_{\mathrm{PSC} . \text { hetero }}=0.53$ with $j s f s=4, \mathrm{P}_{\mathrm{PSC} . \text { hetero }}=0.77$ with $j s f s=7$ and $\mathrm{P}_{\mathrm{PSC} . \text { hetero }}=0.99$ with $j s f s=23$. In contrast, the model of homogeneous migration rates was the most supported, though marginally, in the scenarios of ancient migration (PAM and $\mathrm{AM}$ ): e.g., $\mathrm{P}_{\text {PAM.homo }}=0.56$ with $j s f s=4, \mathrm{P}_{\text {PAM.homo }}=0.69$ with $j s f s=7$ and $\mathrm{P}_{\mathrm{PAM} . h o m o}=0.63$ with $j s f s=23$. Concordant patterns were obtained using the other jSFSs, which differed in the number of individuals and SNPs sampled (Table S2).

Using simulated datasets, we then assessed the performance of the method in identifying the correct model when homogeneous and heterogeneous models were compared (Table S3a). The correct model was always recovered (i.e., an accuracy rate of 1) for the different binnings in all speciation scenarios including gene flow; however, the ambiguity rate did strongly decrease when more information from the jSFS was included. With only four classes $(j s f s=4)$, none of the replicates showed a posterior probability higher than 0.83 (the threshold set for the 2-model comparison), which corresponds to an ambiguity rate of 1 . Similarly, all ambiguity rates were above 0.97 with seven classes $(j s f s=7)$. In contrast, when considering the full jSFS $(j s f s=23)$, we could correctly recover with a strong support the simulated speciation models (e.g., $63 \%$ of the PSC.hetero replicates and $43 \%$ of the PAM.hetero replicates were above the threshold, Table S3a). These results suggest that the additional classes of the 23-binned jSFS are necessary to detect heterogeneity of migration rates across the genome.

\section{Scenarios of speciation}


387 It is clear from Table 3a (see details in Table S1) that binning the jSFS to four or seven classes

388 leads to a loss of information. Specifically, when considering the full jSFS ( $j s f=23)$, only the

389 scenarios involving recent migration were supported $\left(\mathrm{P}_{\mathrm{PSC}}+\mathrm{P}_{\mathrm{SC}}+\mathrm{P}_{\mathrm{IM}}=0.96\right.$, Table $\left.\mathrm{S} 1\right)$; while the

390 contrary was true when fewer classes of polymorphism were used $\left(\mathrm{P}_{\mathrm{PAM}}+\mathrm{P}_{\mathrm{AM}}=0.96\right.$ with $j s f s=4$

391 and 0.93 with $j s f_{s}=7$, Table S1). Remarkably the strict isolation scenario was never supported

$392\left(\mathrm{P}_{\mathrm{SI}}<0.06\right.$, Table $\left.\mathrm{S} 1\right)$ suggesting that gene flow must have occurred between the two mussels

393 species during divergence. Moreover, the fact that the most supported scenario, using full

394 information $(j s f=23)$, was the heterogeneous periodic secondary contact $\left(\mathrm{P}_{\mathrm{PSC} . \text { hetero }}=0.39\right)$ suggests

395 a complex history of speciation, including periods of isolation alternating with ancient and recent

396 migrations. The discrepancies that appear when not distinguishing low and high frequency shared

397 variants (in the case of $j s f s=4$ and $j s f s=7$ ), confirm their importance for the identification of recent

398 migration events (Alcala et al. 2016). In general, the best supported model fits well the data for

399 each binning strategy (Figure S2). All observed statistics were in the $95 \%$ simulated posterior

400 distribution, except for the class "ssfB_2" (derived polymorphism fixed in species B, but

401 polymorphic (doubletons) in species A) which was slightly overestimated by the model

402 PSC.hetero $(j s f s=23$, Figure S2a); and the class "sfB" (derived polymorphism fixed in species B,

403 and absent in species A) which was slightly underestimated by the model PAM.hetero (jsfs=7,

404 Figure S2b). No statistics were found significantly out of the simulated distribution under model

405 PAM.hetero with $j s f s=4$ (Figure S2c). On the contrary, the alternative models failed to fit more

406 parts of the jSFS. For example with $j s f s=23$ (Figure S2a), in which PSC.hetero is the best model

$407\left(\mathrm{P}_{\mathrm{PSC}}=0.385\right)$, the alternative model PAM.hetero $\left(\mathrm{P}_{\mathrm{PAM}}=0.007\right)$ overestimates the number of

408 derived sites fixed within each species in all 'sf' classes and model SI $\left(\mathrm{P}_{\mathrm{SI}}=0.009\right)$ underestimates

409 the number of shared polymorphisms in most 'ss' classes.

410 We further evaluate, by simulation, the effect of binning the jSFS on the capacity of the method

411 to infer the correct speciation model (Table S3b). As the heterogeneous models consistently 
412 outperformed the homogeneous models in scenarios with ongoing migration, and they were not 413 significantly less likely in the models of ancient migration (Table S2), the ABC performance was 414 evaluated among the five models of migration including heterogeneous migration only 415 (IM.hetero, SC.hetero, PSC.hetero, AM.hetero and PAM.hetero), plus the strict isolation model 416 (SI). Globally, the probability of rejecting the correct model decreased when increasing the 417 number of polymorphic classes. By using four or seven classes vs. twenty-three classes, the 418 correct model was recovered at an estimated rate equal to or lower than 0.70 vs. 1 for IM.hetero, 4190.58 vs. 0.50 for SC.hetero, 0.89 vs. 1 for PSC.hetero, 0.34 vs. 0.42 for AM.hetero, 0.70 vs. 0.79 420 for PAM.hetero and 0.73 vs. 0.84 for SI (Table S3b). Moreover, we could not discriminate 421 between the different scenarios of recent migration (PSC.hetero, SC.hetero and IM.hetero) when 422 using only four or seven classes (Figure S3a); and the same was true with the two scenarios of 423 ancient migration (PAM.hetero and AM.hetero, Figure S3b). In contrast, the full jSFS $(j s f s=23)$ 424 contains enough information to more accurately identify the periodic secondary contact as the 425 true model among the other scenarios of recent migration (Figure S3a). Nevertheless, 426 distinguishing between the two ancient migration scenarios remained difficult even with $j s f s=23$ 427 (Figure S3b). Finally, the ambiguity rates also strongly decreased when binning the jSFS in more 428 classes: all ambiguity rates were equal to or greater than 0.86 in the recent migration scenarios, 4290.41 in the ancient migration scenarios and 0.21 in the SI scenario with $j s f s=4$ or $j s f s=7$; whereas 430 these numbers were $0.25,0.20$ and 0.15 with $j s f s=23$ (Table $\mathrm{S} 3 \mathrm{~b}$ ).

\section{$\underline{\text { Discussion }}$}

434 NGS data give us the opportunity to capture the diversity of coalescent histories across loci, and 435 so to reveal the complexity of the speciation process (Sousa \& Hey 2013). Recently, important 
436 efforts have been made to develop statistical methods of inference making use of population

437 genomics data. Computing the jSFS is an efficient way of summarizing the demographic

438 information contained in NGS data because anonymous SNPs can be used (e.g. produced by

439 RAD-sequencing) and it does not rely on phased data. However, the jSFS obtained from low-

440 coverage sequencing data (typically $<10 \mathrm{x}$ per position and per individual) can be biased toward a

441 deficit of rare variants; and this is of particular concern when investigating the demographic

442 history of populations (e.g., Nielsen et al. 2012; Han et al. 2013). A first category of maximum-

443 likelihood methods uses forward diffusion theory to compute numerical solutions to the jSFS

444 under complex models (see Gutenkunst et al. 2009; Lukic \& Hey 2012). A second category of

445 methods estimates the expected jSFS under any demographic model, as simulations are used to

446 approximate the composite-likelihood of the data (see Naduvilezhath et al. 2011; Excoffier et al.

447 2013). Here, we used a likelihood-free method (ABC) on the jSFS of coding sequences, and we

448 evaluated the influence of different sampling strategies on the inference of speciation models in

449 mussels.

450 Our ABC-based model comparison shows little qualitative effect of individual and SNP sampling

451 on the outcomes. First, inferences based on $n=2, n=4$ or $n=8$ individuals supported the same

452 model of periodic secondary contact (Table 3), consistent with previous results that relatively few

453 individuals are sufficient to make robust inferences on the way divergence occurs between

454 lineages (e.g., Robinson et al. 2014). This is because most coalescence events occur recently in

455 the population history; so increasing the number of individuals is only helpful to characterize

456 recent demographic events rather than the past divergence history. Second, we showed that

457 inferences from the two sequencing techniques ("exome capture" vs. "rna-seq") were

458 qualitatively the same, despite the very different number of SNPs that were sampled, again

459 supporting the periodic secondary contact scenario (Table 3). This implies that neither the

460 sequencing technique, nor the number of informative sites have a substantial effect on the 
461 inference. In fact, the jSFS calculated from the different data sets were very similar (Figure S1);

462 we only found a deficit of divergent SNPs in the "exome capture" data that may be due to the

463 difficulty of mapping highly divergent alleles onto a single reference (on the contrary, the "rna-

464 seq" reads from the different species were independently assembled and mapped). From a

465 theoretical perspective, adding more loci (or longer loci) provide information about deep

466 coalescent events which are important for shedding light on the divergence history of closely-

467 related species (e.g., Wang \& Hey 2010). In fact, previous simulation studies showed an

468 influence of locus length and locus number on the $\mathrm{ABC}$ performance in model choice, and

469 highlighted a threshold effect above which adding more loci did not significantly improve

470 inferences (e.g., Li \& Jakobsson 2012; Robinson et al. 2014; Shafer et al. 2015). Thereby, we

471 argue that the number of SNPs sampled in our study was sufficient to accurately represent the

472 diversity of coalescent histories in the Mytilus genome, and consistently support the same 473 speciation model.

474 In most studies, functions of the jSFS are used as summaries of the data. For example, the 475 likelihood method of Nielsen \& Wakeley (2001) uses four classes of polymorphisms to estimate 476 migration rates and divergence times in the isolation with migration scenario. In the $\mathrm{ABC}$ 477 approach, choosing a suitable set of summary statistics is difficult because it implies a trade-off 478 between loss of information and reduction of dimensionality. Accordingly, practical methods to 479 identify approximately sufficient statistics have been developed (e.g., Wegmann et al. 2009; 480 Nunes \& Balding 2010; Aeschbacher et al. 2012). Here, we compared ABC inferences based on 481 23-binned jSFS $(j s f s=23)$ with inferences using a subset of polymorphic classes $(j s f s=4$ and $\left.482 j s f s_{s}=7\right)$. Model checkings through simulations pointed out the loss of information when only four 483 or seven classes in the jSFS were considered; particularly for the inference of recent migration 484 events. By decomposing the jSFS into twenty-three classes, we could reveal the excess of shared 485 polymorphisms that are at high frequency in one species and low frequency in the other, a pattern 
486 produced by recent migrants (Table 3). This is in agreement with the simulation study of Tellier

487 et al. (2011) that showed a significant improvement in the estimation of the timing of gene flow

488 when these additional polymorphic classes were considered; and the study of Alcala et al. (2016)

489 that showed an excess of high frequency derived alleles is the characteristic footprint of

490 secondary contacts. Across all models, we showed that the probability to correctly infer the true

491 model (accuracy rate) increases with the number of classes considered, and the ambiguity rate

492 correspondingly decreases (Table S3). Smith et al. (2017) similarly found that the statistical

493 power of their $\mathrm{ABC}$ model selection increases with the number of classes until reaching a plateau

494 of error rates (but above which computation efforts continues to increase).

495 Inferences based on the 23-bin jSFS consistently support a model of periodic secondary contact

496 with genomic heterogeneity in gene flow (PSC.hetero) in both the "exome capture" and "rna-seq"

497 mussel datasets, and simulations showed that this model has a high accuracy rate, and one of the

498 lowest ambiguity rates (Table S3). Although its relative posterior probability was moderately

499 higher than that of the other models with migration (SC and IM, Table S1), we showed that the

500 method has some power to distinguish PSC from these models (Figure S3a). These results are in

501 agreement with previous ABC-based studies revealing clearly a secondary contact history

502 between the two mussel species (Fraïsse et al. 2014; Roux et al. 2014), although they relied on

503 eight nuclear loci and on a different set of summary statistics (those that we called "mscalc"

504 here). The periodic connectivity models (PSC and PAM) were not included in these previous

505 studies because of the lack of power to test for intermittent gene exchange since secondary

506 contact. In the present study, we directly compared the use of the jSFS vs. "mscalc" statistics by

507 performing additional $\mathrm{ABC}$ inferences on the "mscalc" statistics presented in Table 2. Results

508 were similar to those obtained with $j s f s=23$ (Table S1), i.e. the best supported models included

509 one or two secondary contacts, except for two datasets ("exome capture" data with $n=2$ and $n=8$

510 individuals) for which strict isolation was chosen with a posterior probability of $37 \%$ and $34 \%$, 
511 respectively. However, the goodness-of-fit of the strict isolation model was quite poor for the

512 standard deviation of the number of fixed sites, "sf_std", and very poor for the standard deviation

513 of the FST, 'fst_std' ("exome capture" data with $n=2$ individuals, Figure S2d). Both statistics

514 were underestimated by the model suggesting that a history without gene flow cannot produce the

515 observed variation of genetic divergence along the genome. Across models, inferences based on

516 the "mscalc" statistics showed similar accuracy rate to those based on $j s f_{s}=23$; however, the

517 ambiguity rates for models with current migration were somewhat higher (PSC $=0.52$ vs. 0.30,

$518 \mathrm{SC}=0.61$ vs. $0.31, \mathrm{IM}=0.40$ vs. 0.25 , respectively). These supplementary analyses suggest that

519 extracting summary statistics from the jSFS can lead to a substantial loss of information.

520

521

\section{Conclusion}

523 In this work, we have shown that two high-throughput sequencing datasets ("exome capture" and

524 "rna-seq"), imply the same history of divergence in mussels, regardless of the number of

525 individuals or SNPs sampled, but conditional on the inclusion of informative classes in the jSFS.

526 Thus, genome-wide data coupled with flexible inference methods allow us to test for more

527 complex scenarios of divergence, by providing a comprehensive picture of the gene histories

528 across the genome. Here, we incorporate in our ABC framework heterogeneity in migration rates

529 among loci, to account for the semi-permeability of the barrier to gene flow between recently

530 diverged species (Barton \& Bengtsson 1986). This variation in the rate of effective migration

531 results in variation of genetic divergence along the genome. As shown elsewhere (Roux et al.

532 2013, 2014, 2016), failing to account for this heterogeneity can mislead inferences. In a similar

533 way, the effect of background selection (i.e. purifying selection at linked loci) and genetic

534 hitchhiking (i.e. positive selection at linked loci) in regions of low recombination can now be 
535 incorporated in demographic inferences by including heterogeneity in effective sizes among loci

536 (Sousa \& Hey 2013, Roux et al. 2016, Aeschbacher et al. 2017). Combining modelling

537 approaches that account for both sources of genomic heterogeneity (e.g., Roux et al. 2016) may

538 provide further insight into the complex interplay between linked selection and resistance to

539 introgression during speciation with gene flow (e.g., Duranton et al. 2018).

540

\section{Acknowledgements}

542 The computations were performed at the "Montpellier Bioinformatics Biodiversity" computing

543 cluster platform (CeMEB LabEx "Mediterranean Center for Environment and Biodiversity").

544 This is article XXX of "Institut des Sciences de l'Evolution de Montpellier".

545

546 Data Accessibility

547 Data set 1: "exome capture": http://www.scbi.uma.es/mytilus/index.php (Fraïsse et al. 2016).

548 Data set 2: "rna-seq": http://kimura.univ-montp2.fr/PopPhyl (Romiguier et al. 2014).

550 References

551

552

- Aeschbacher S, Selby JP, Willis JH, Coop G (2017) Population-genomic inference of the strength and timing of selection against gene flow. Proc. Natl. Acad. Sci. USA, 114(27), 7061-7066.

- Aeschbacher S, Beaumont MA, Futschik A (2012) A novel approach for choosing summary statistics in approximate Bayesian computation. Genetics, 192(3), 1027-1047.

- Alcala N, Jensen JD, Telenti, A, Vuilleumier S (2016) The genomic signature of population reconnection following isolation: from theory to HIV. G3(Bethesda), 6(1), 107-120.

- Andolfatto, P. (2005). Adaptive evolution of non-coding DNA in Drosophila. Nature, 437(7062), 1149. 
- Barton N, Bengtsson BO (1986) The barrier to genetic exchange between hybridising populations. Heredity, 57(3), 357-376.

568

569

570

571

572

573

574

575

576

577

578

579

580

581

582

583

584

585

586

587

588

589

590

591

592

593

594

595

596

597

598

599

600

601

602

603

604

- Beaumont MA, Nielsen R, Robert C, Hey J, Gaggiotti O, Knowles L, Estoup A, Panchal M, Corander J, Hickerson M, Sisson SA, Fagundes N, Chikhi L, Beerli P, Vitalis R, Cornuet JM, Huelsenbeck J, Foll M, Yang Z, Rousset F, Balding D, Excoffier L. (2010) In defence of model-based inference in phylogeography. Mol. Ecol., 19(3), 436-446.

- Beaumont MA, Zhang W, Balding DJ (2002) Approximate Bayesian computation in population genetics. Genetics, 162(4), 2025-2035.

- Becquet C, Przeworski M (2007) A new approach to estimate parameters of speciation models with application to apes. Genome Res., 17(10), 1505-1519.

- Bierne N, Borsa P, Daguin C, Jollivet D, Viard F, Bonhomme F, David P (2003a) Introgression patterns in the mosaic hybrid zone between Mytilus edulis and M. galloprovincialis. Mol. Ecol., 12(2), 447-461.

- Bierne N, Bonhomme F, David P (2003b) Habitat preference and the marine-speciation paradox. Proc. R. Soc. B, 270(1522), 1399-1406.

- Bierne N, David P, Boudry P, Bonhomme F (2002) Assortative fertilization and selection at larval stage in the mussels Mytilus edulis and M. galloprovincialis. Evolution, 56(2), 292-298.

- Boitard S, Rodríguez W, Jay F, Mona S, Austerlitz F (2016) Inferring population size history from large samples of genome-wide molecular data - An approximate Bayesian Computation approach. PLoS Genet., 12(3), e1005877.

- Boon E, Faure MF, Bierne N (2009) The flow of antimicrobial peptide genes through a genetic barrier between Mytilus edulis and M. galloprovincialis. J. Mol. Evol., 68(5), 461474.

- Cabrera AA, Palsbøll PJ (2017) Inferring past demographic changes from contemporary genetic data: a simulation $\square$ based evaluation of the $\mathrm{ABC}$ methods implemented in DIYABC. Mol Ecol Resour., 17:e94-e110.

- Cahais V, Gayral P, Tsagkogeorga G, Melo $\square$ Ferreira J, Ballenghien M, Weinert L, Chiari Y, Belkhir K, Ranwez V, Galtier N (2012) Reference $\square$ free transcriptome assembly in 
605 non $\square$ model animals from next $\square$ generation sequencing data. Mol. Ecol. Resour., 12(5), $606 \quad 834-845$.

607

608 - Chapman MA, Hiscock SJ, Filatov DA (2013) Genomic divergence during speciation

609

610 driven by adaptation to altitude. Mol. Biol. Evol., 30(12), 2553-2567.

- Christe C, Stölting KN, Paris M, Fraïsse C, Bierne N, Lexer C (2017) Adaptive evolution and segregating load contribute to the genomic landscape of divergence in two tree species connected by episodic gene flow. Mol. Ecol., 26(1), 59-76.

- Csilléry K, François O, Blum MGB (2012) abc: an R package for approximate Bayesian computation (ABC). Methods Ecol. Evol., 3(3), 475-479.

- Domingues VS, Poh YP, Peterson BK, Pennings PS, Jensen JD, Hoekstra HE (2012) Evidence of adaptation from ancestral variation in young populations of beach mice. Evolution, 66(10), 3209-3223.

- Excoffier L, Dupanloup I, Huerta-Sánchez E, Sousa VC, Foll M (2013) Robust demographic inference from genomic and SNP data. PLoS Genet., 9(10), e1003905.

- Fagundes NJ, Ray N, Beaumont M, Neuenschwander S, Salzano FM, Bonatto SL, Excoffier L (2007) Statistical evaluation of alternative models of human evolution. Proc. Natl. Acad. Sci. USA, 104(45), 17614-17619.

- Fraïsse C, Belkhir K, Welch JJ, Bierne N (2016) Local interspecies introgression is the main cause of extreme levels of intraspecific differentiation in mussels. Mol. Ecol., 25(1), $269-286$.

- Fraïsse C, Roux C, Welch JJ, Bierne N (2014) Gene-flow in a mosaic hybrid zone: is local introgression adaptive? Genetics, 197(3), 939-951.

- Gayral P, Melo-Ferreira J, Glémin S, Bierne N, Carneiro M, Nabholz B, Lourenco JM, Alves PC, Ballenghien M, Faivre N, Belkhir K, Cahais V, Loire E, Bernard A, Galtier N (2013) Reference-free population genomics from next-generation transcriptome data and the vertebrate-invertebrate gap. PLoS Genet., 9(4), e1003457.

- Gutenkunst RN, Hernandez RD, Williamson SH, Bustamante CD (2009) Inferring the joint demographic history of multiple populations from multidimensional SNP frequency data. PLoS Genet., 5(10), e1000695. 
- Han E, Sinsheimer JS, Novembre J (2013) Characterizing bias in population genetic

646

647

648

649

650

651

652

653

654

655

656

657

658

659

660

661

662

663

664

665

666

667

668

669

670

671

672

673

674

675

676

677

678

679

680

681

682

683

684 inferences from low-coverage sequencing data. Mol. Biol. Evol., 31(3), 723-735.

- Hewitt G (2000) The genetic legacy of the Quaternary ice ages. Nature, 405(6789), 907913.

- Hey J, Nielsen R (2007) Integration within the Felsenstein equation for improved Markov chain Monte Carlo methods in population genetics. Proc. Natl. Acad. Sci. USA, 104(8), $2785-2790$.

- Hudson RR (2002) Generating samples under a Wright-Fisher neutral model of genetic variation. Bioinformatics, 18(2), 337-338.

- Li H, Durbin R (2009) Fast and accurate short read alignment with Burrows-Wheeler transform. Bioinformatics, 25(14), 1754-1760.

- Li S, Jakobsson M (2012) Estimating demographic parameters from large-scale population genomic data using Approximate Bayesian Computation. BMC Genet., 13(1), 22.

- Li X, Jian Y, Xie C, Wu J, Xu Y, Zou C (2017) Fast diffusion of domesticated maize to temperate zones. Sci. Rep., 7(1), 2077.

- Lukic S, Hey J (2012) Demographic inference using spectral methods on SNP data, with an analysis of the human out-of-Africa expansion. Genetics, 192(2), 619-639.

- Marino IAM, Benazzo A, Agostini C, Mezzavilla M, Hoban SM, Patarnello T, Zane L, Bertorelle G (2013) Evidence for past and present hybridization in three Antarctic icefish species provides new perspectives on an evolutionary radiation. Mol. Ecol., 22(20), 51485161.

- McCoy RC, Garud NR, Kelley JL, Boggs CL, Petrov DA (2014) Genomic inference accurately predicts the timing and severity of a recent bottleneck in a nonmodel insect population. Mol. Ecol., 23(1), 136-150.

- Naduvilezhath L, Rose LE, Metzler D (2011) Jaatha: a fast composite-likelihood approach to estimate demographic parameters. Mol. Ecol., 20(13), 2709-2723.

- Nielsen R, Korneliussen T, Albrechtsen A, Li Y, Wang J (2012) SNP calling, genotype calling, and sample allele frequency estimation from New-Generation Sequencing data. 
685

686

687

688

689

690

691

692

693

694

695

696

697

698

699

700

701

702

703

704

705

706

707

708

709

710

711

712

713

714

715

716

717

718

719

720

721

722

723

PloS One, 7(7), e37558.

- Nielsen R, Wakeley J (2001) Distinguishing migration from isolation: a Markov chain Monte Carlo approach. Genetics, 158(2), 885-896.

- Nunes MA, Balding DJ (2010) On optimal selection of summary statistics for approximate Bayesian computation. Stat Appl Genet Mol Biol., 9(1).

- Qi X, An H, Ragsdale AP, Hall TE, Gutenkunst RN, Chris Pires J, Barker MS (2017) Genomic inferences of domestication events are corroborated by written records in Brassica rapa. Mol. Ecol., 26(13), 3373-3388.

- Ramos-Onsins SE, Stranger BE, Mitchell-Olds T, Aguadé M (2004) Multilocus analysis of variation and speciation in the closely related species Arabidopsis halleri and A. lyrata. Genetics, 166(1), 373-388.

- Robinson JD, Bunnefeld L, Hearn J, Stone GN, Hickerson MJ (2014) ABC inference of multi-population divergence with admixture from unphased population genomic data. Mol. Ecol., 23(18), 4458-4471.

- Romiguier J, Gayral P, Ballenghien M, Bernard A, Cahais V, Chenuil A, Chiari Y, Dernat R, Duret L, Faivre N, Loire E, Lourenco JM, Nabholz B, Roux C, Tsagkogeorga G, Weber AA, Weinert LA, Belkhir K, Bierne N, Glémin S, Galtier N (2014) Comparative population genomics in animals uncovers the determinants of genetic diversity. Nature, 515(7526), 261-263.

- Ross-Ibarra J, Wright SI, Foxe JP, Kawabe A, DeRose-Wilson L, Gos G, Charlesworth D, Gaut BS (2008) Patterns of polymorphism and demographic history in natural populations of Arabidopsis lyrata. PloS One, 3(6), e2411.

- Rougeux C, Bernatchez L, Gagnaire P-A (2017) Modeling the multiple facets of speciation-with-gene-flow toward inferring the divergence history of lake Whitefish species pairs (Coregonus clupeaformis). Genome Biol. Evol., 9(8), 2057-2074.

- Roux C, Fraïsse C, Romiguier J, Anciaux Y, Galtier N, Bierne N (2016) Shedding light on the grey zone of speciation along a continuum of genomic divergence. PLoS Biol., 14(12), e2000234.

- Roux C, Fraïsse C, Castric V, Vekemans X, Pogson GH, Bierne N (2014) Can we 
724

725

726

727

728

729

730

731

732

733

734

735

736

737

738

739

740

741

742

743

744

745

746

747

748

749

750

751

752

753

754

755

756

757

758

759

760

761

762

763 continue to neglect genomic variation in introgression rates when inferring the history of speciation? A case study in a Mytilus hybrid zone. J. Evol. Biol., 27(8), 1662-1675.

- Roux C, Tsagkogeorga G, Bierne N, Galtier N (2013) Crossing the species barrier: genomic hotspots of introgression between two highly divergent Ciona intestinalis species. Mol. Biol. Evol., 30(7), 1574-1587.

- Roux C, Castric V, Pauwels M, Wright SI, Saumitou-Laprade P, Vekemans X (2011) Does speciation between Arabidopsis halleri and Arabidopsis lyrata coincide with major changes in a molecular target of adaptation? PloS One, 6(11), e26872.

- Shafer ABA, Gattepaille LM, Stewart REA, Wolf JBW (2015) Demographic inferences using short-read genomic data in an approximate Bayesian computation framework: in silico evaluation of power, biases and proof of concept in Atlantic walrus. Mol. Ecol., 24(2), 328-345.

- Simon A, Bierne B, Welch JJ (2017) Coadapted genomes and selection on hybrids: Fisher's geometric model explains a variety of empirical patterns. bioRxiv 237925; doi: https://doi.org/10.1101/237925

- Skibinski DOF, Beardmore JA, Cross TF (1983) Aspects of the population genetics of Mytilus (Mytilidae; Mollusca) in the British Isles. Biol. J. Linn. Soc., 19(2), 137-183.

- Smith ML, Ruffley M, Espíndola A, Tank DC, Sullivan J, Carstens BC (2017) Demographic model selection using random forests and the site frequency spectrum. Mol. Ecol., 26, 4562-4573.

- Sousa VC, Carneiro M, Ferrand N, Hey J (2013) Identifying loci under selection against gene flow in isolation-with-migration models. Genetics, 194(1), 211-233.

- Sousa V, Hey J (2013) Understanding the origin of species with genome-scale data: modelling gene flow. Nature Rev. Genet., 14(6), 404-414.

- Tajima, F. (1989a) DNA polymorphism in a subdivided population: the expected number of segregating sites in the two-subpopulation model. Genetics, 123(1), 229-240.

- Tajima, F. (1989b) The effect of change in population size on DNA polymorphism. Genetics, 123(3), 597-601.

- Tajima F (1983) Evolutionary relationship of DNA sequences in finite populations. 
764

765

766

767

768

769

770

771

772

773

774

775

776

777

778

779

780

781

782

783

784

785

786

787

788

789

790

791

792

793

794

795

796

797

Genetics, 105(2), 437-460.

- Tellier A, Pfaffelhuber P, Haubold B, Naduvilezhath L, Rose L, Städler T, Stephan W, Metzler D (2011) Estimating parameters of speciation models based on refined summaries of the joint site-frequency spectrum. PloS One, 6(5), e18155.

- Tine M, Kuhl H, Gagnaire P-A, Louro B, Desmarais E (2014) European sea bass genome and its variation provide insights into adaptation to euryhalinity and speciation. Nature Comm., 5, 5770 .

- Tsagkogeorga G, Cahais V, Galtier N (2012) The population genomics of a fast evolver: high levels of diversity, functional constraint, and molecular adaptation in the tunicate Ciona intestinalis. Genome Biol. Evol., 4(8), 740-749.

- Wakeley J, Hey J (1997) Estimating ancestral population parameters. Genetics, 145(3), $847-855$.

- Wang Y, Hey J (2010) Estimating divergence parameters with small samples from a large number of loci. Genetics, 184(2), 363-379.

- Watterson GA (1975) On the number of segregating sites in genetical models without recombination. Theor. Popul. Biol., 7(2), 256-276.

- Wegmann D, Leuenberger C, Excoffier L (2009) Efficient approximate Bayesian computation coupled with Markov chain Monte Carlo without likelihood. Genetics, 182(4), 1207-1218.

- Wu C-I (2001) The genic view of the process of speciation. J. Evol. Biol., 14(6), 851-865.

- Xue AT, Hickerson MJ (2015) The aggregate site frequency spectrum for comparative population genomic inference. Mol. Ecol., 24(24), 6223-6240. 
798 Figures

799 Figure 1. Models of speciation.

800 Figure 2. Decomposition of the unfolded jSFS for $n=2$ individuals (i.e. 4 alleles) in each species. 801

802 Tables

803 Table 1. Sampling design.

804 Table 2. Summary statistics (mscalc).

805 Table 3. Posterior probabilities of the speciation models.

806

\section{Supplementary Figures}

808 Figure S1. Unfolded jSFS for the two different sequencing techniques.

809 Figure S2. Goodness-of-fit test for three models (PSC, PAM and SI) with the "exome capture" 810 data, $n=2$ individuals and the different summary statistics.

811 Figure S3. Evaluation of the ABC performance on model choice.

812

813 Supplementary Tables

814 Table S1. Posterior probabilities - 11 models.

815 Table S2. Posterior probabilities - homo vs. hetero.

816 Table S3. Accuracy and ambiguity rate ( $n=2$, "exome capture" data).

818 Supplementary Methods

819 Text S1. Scripts to compute summary statistics from the data. a. Extracts polymorphic 820 synonymous positions from a fasta alignment. b. Produces the jSFS for each contig, and mscalc 821 statistics across contigs. c. Produces a summed jSFS across contigs.

822 Text S2. Scripts to compute summary statistics from simulations. a. Produces coalescent 823 simulations under a set of demographic models, and compute jSFS for each contig, and mscalc 824 statistics across contigs. b. Produces a summed jSFS across contigs.

825 Text S3. Scripts to estimate the posterior probability of each demographic model using neural 826 networks based on jSFS (a) and mscalc statistics (b). Scripts to evaluate the ability of the method 827 to correctly recover the true simulated model based on jSFS (c) and mscalc statistics (d). 


\section{Figure 1 (on next page)}

\section{Models of speciation}

Six classes of scenarios with different temporal patterns of migration are compared (left column); and for those including migration, two versions are depicted assuming either homogeneity ("homo") or heterogeneity ("hetero") of effective migration rate across the genome (right column). All scenarios assume that an ancestral population of effective size $N_{\mathrm{A}}$ split $T_{\text {split }}$ generations ago into two populations of constant sizes $N_{1}$ and $N_{2}$. At the two extremes, divergence occurs in allopatry (SI, strict isolation) or under continuous migration (IM, isolation with migration). Through time, migration occurs at a constant rate $M_{12}$ from population 1 to population 2 and $M_{21}$ in the opposite direction. Ancient migration (AM) and periodic ancient migration (PAM) scenarios both assume that populations started diverging in the presence of gene flow. Then they experienced a single period of isolation, $T_{\text {iso, }}$ in the AM model while intermittent gene flow occurred in the PAM model. In the secondary contact (SC) and periodic secondary contact (PSC) scenarios, populations diverged in the absence of gene flow followed by a single period of secondary contact, $T_{s c}$, in the SC model while intermittent gene flow occurred in the PSC model. 


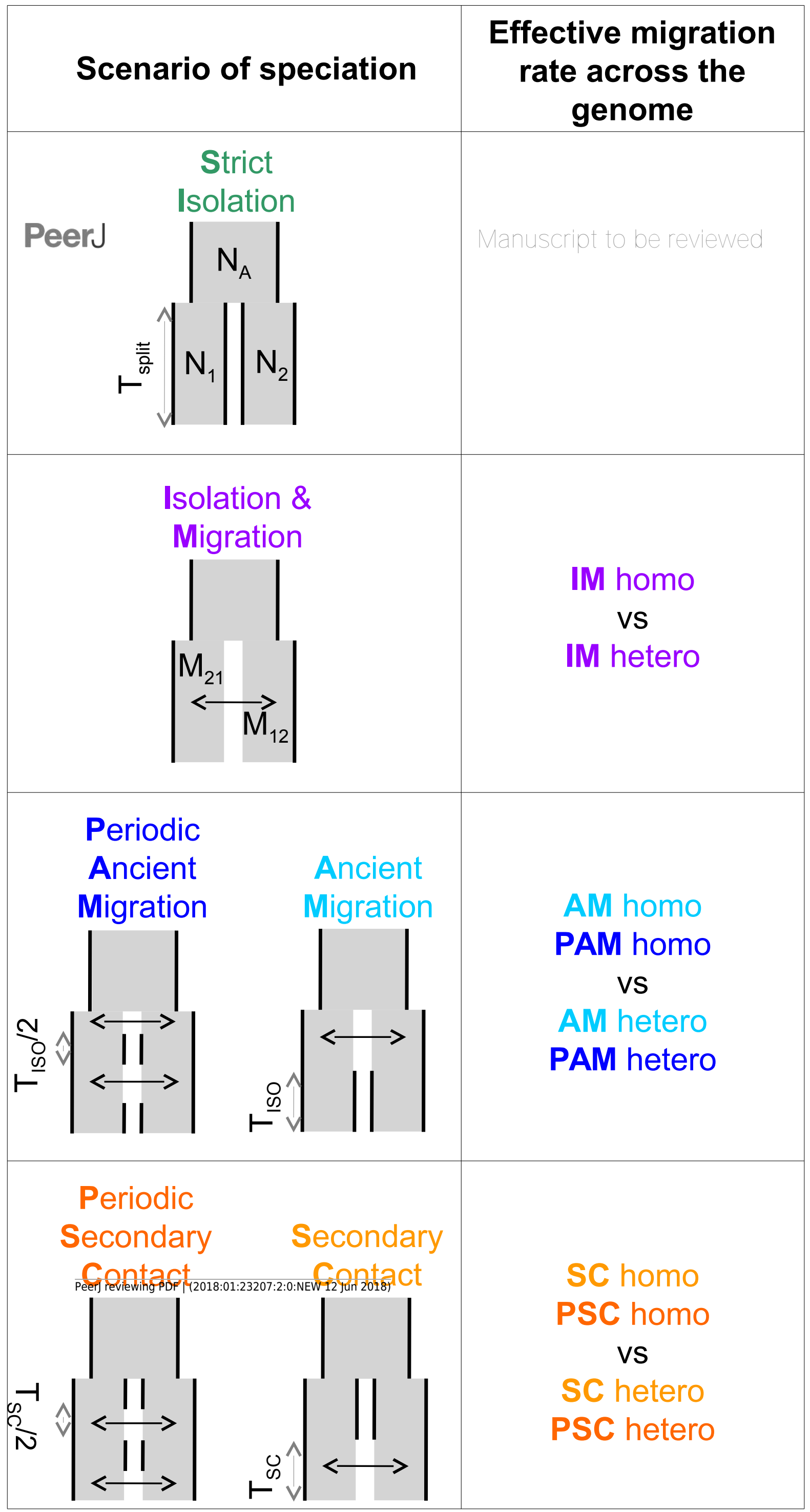




\section{Figure 2 (on next page)}

Decomposition of the unfolded joint site frequency spectrum for $n=2$ individuals (i.e. 4 alleles) in each species

The density of derived alleles in species 1 ( $M$. edulis, $x$ axis) and species 2 ( $M$.

galloprovincialis, y axis) is indicated by a number within each cell. Only sites showing two distinct alleles in the inter-specific alignment were considered, hence the cells $\{0 ; 0\}$ and $\{4 ; 4\}$ have been masked. The total number of polymorphic sites is 3,993 SNPs ("exome capture" data). (A) Decomposition of the jSFS into four classes of polymorphism without an outgroup sequence (i.e., the Wakeley-Hey classes): fixed differences (black), private polymorphisms in species 1 (blue) or species 2 (red) and shared polymorphisms (green).

Decomposition of the jSFS into seven classes of polymorphism by using the sequenced outgroup. Two alleles are differentially fixed between the two species: the derived allele can be fixed in species 1 (black) or in species 2 (grey). Exclusive polymorphism can be the result of a recent mutation specific to species 1 (blue) or species 2 (red); but it can also be the result of an ancestral mutation only fixed in species 2 (cyan) or in species 1 (orange). Shared polymorphisms are shown in green. (C) Decomposition of jSFS into twenty-three classes of polymorphism. Singletons and doubletons in each species were included as new classes. Note that in the case of $n=2$, this is the full spectrum. 
A.

jsfs $=4$

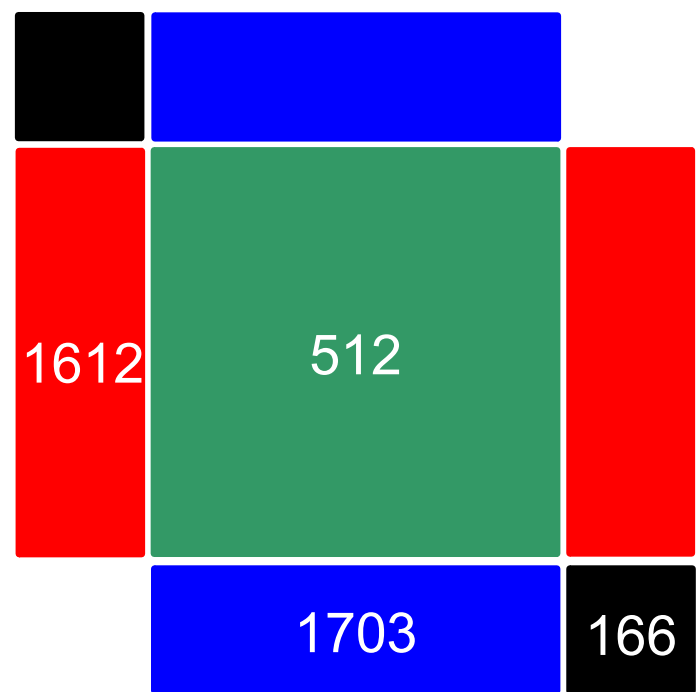

B PeerJ

jsfs $=7$

\begin{tabular}{|c|c|}
\hline 104 & 196 \\
\hline 1401 & 512 \\
\hline & 1507 \\
\hline
\end{tabular}

$$
\text { jsfs }=23
$$

\begin{tabular}{|c|c|c|c|c|c|}
\hline 104 & 38 & 30 & 128 & & 4 \\
\hline 95 & 45 & 26 & 36 & 85 & \\
\hline 217 & 72 & 32 & 21 & 64 & \\
\hline 1089 & 182 & 58 & 40 & 62 & \\
\hline & 1299 & 145 & 63 & 62 & 0 \\
\hline 0 & 1 & $\begin{array}{c}2 \\
\text { edul }\end{array}$ & 3 & 4 & \\
\hline
\end{tabular}




\section{Table $\mathbf{1}$ (on next page)}

Sampling design

technique: rna sequencing ("rna-seq") vs. exome enrichment sequencing ("exome capture"); $n$ : number of individuals sampled in each population. 
M. edulis M. galloprovincialis

technique

$\mathrm{n}$

population

locality

Wadden Sea, Holland

Lupin/Fouras, France

Brittany

Mediterranean Sea

Roscoff, France

Sète, France

Brittany

Mediterranean Sea
Roscoff, France

Sète, France
M. trossulus (outgroup)

population locality

population locality

Europe

Tvärminne, Finland

Tvärminne, Finland

Europe

Seattle, USA

Seattle, USA

USA Seattle, USA




\section{Table 2 (on next page)}

\section{Summary statistics (mscalc)}

technique: rna sequencing ("rna-seq") vs. exome enrichment sequencing ("exome capture"); $n$ : number of individuals analyzed in each species; $n_{\text {locus: }}$ total number of locus $n_{S N P}$ : total number of polymorphic sites.

The following statistics were calculated for each locus. Their average (in black) and standard deviation (in grey) across all loci are given.

$S$ : number of polymorphic sites; $S f$ : number of fixed differences; $S x$ : number of exclusive polymorphic sites; Ss: number of shared polymorphic sites; $\pi$ : number of pairwise differences (Tajima, 1983); $\theta_{w}$ : Watterson's $\theta$ (Watterson, 1975); D: Tajima's D (Tajima, 1989a, 1989b); FST $=1-\pi_{S} / \pi_{T}$ : level of species differentiation, where $\pi_{S}$ is the average pairwise nucleotide diversity within species and $\pi_{T}$ is the total pairwise nucleotide diversity of the pooled sample across species; div: total interspecific divergence; netdiv: net molecular divergence measured at synonymous positions. 
technique $\quad \mathrm{n}_{1} \quad \mathrm{n}_{2} \quad \mathrm{n}_{\text {locus }} \quad \mathrm{n}_{\mathrm{SNP}} \quad \mathrm{S} \quad \mathrm{S} \_\mathrm{sd} \quad \mathrm{Sf} \quad \mathrm{Sf}$ sd $\quad \mathrm{Sx}_{1} \quad \mathrm{Sx}_{1-} \mathrm{sd} \quad \mathrm{Sx}_{2} \quad \mathrm{Sx}_{2-} \mathrm{sd} \quad \mathrm{Ss} \quad \mathrm{Ss} \_\mathrm{sd}$

\begin{tabular}{|c|c|c|c|c|c|c|c|c|c|c|c|c|c|c|}
\hline \multirow{3}{*}{$\begin{array}{l}\text { exome } \\
\text { capture }\end{array}$} & 2 & 2 & 516 & 3993 & 7.738 & 6.732 & 0.322 & 1.285 & 3.124 & 3.077 & 3.3 & 3.509 & 0.992 & 1.855 \\
\hline & 4 & 4 & 557 & 5092 & 9.142 & 8.076 & 0.097 & 0.583 & 3.555 & 3.434 & 3.896 & 4.006 & 1.594 & 2.482 \\
\hline & 8 & 8 & 512 & 5000 & 9.766 & 8.828 & 0.025 & 0.296 & 3.504 & 3.502 & 4.258 & 4.363 & 1.979 & 2.761 \\
\hline \multirow{2}{*}{ rna-seq } & 2 & 2 & 2147 & 17275 & 8.046 & 6.554 & 0.81 & 3.057 & 2.966 & 3.14 & 2.809 & 2.851 & 1.462 & 2.216 \\
\hline & 4 & 4 & 1842 & 17902 & 9.719 & 7.953 & 0.507 & 2.608 & 3.344 & 3.328 & 3.368 & 3.318 & 2.501 & 3.318 \\
\hline
\end{tabular}

$\pi_{1} \quad \pi_{1-} \mathrm{sd} \quad \pi_{2} \quad \pi_{2-} \mathrm{sd} \quad \theta_{\mathrm{w} 1} \quad \theta_{\mathrm{w} 1-} \mathrm{sd} \quad \theta_{\mathrm{w} 2} \quad \theta_{\mathrm{w} 2-} \mathrm{sd} \quad \mathrm{D}_{1} \quad \mathrm{D}_{1-} \mathrm{sd} \quad \mathrm{D}_{2} \quad \mathrm{D}_{2-} \mathrm{sd} \quad \mathrm{FST} \quad \mathrm{FST}$ sd div $\quad$ div_sd netdiv netdiv_sd

$\begin{array}{lllllllllllllllllll}0.016 & 0.016 & 0.016 & 0.015 & 0.017 & 0.017 & 0.016 & 0.016 & -0.27 & 0.674 & -0.391 & 0.513 & 0.114 & 0.189 & 0.02 & 0.017 & 0.004 & 0.009 \\ 0.014 & 0.014 & 0.013 & 0.013 & 0.016 & 0.015 & 0.016 & 0.015 & -0.668 & 0.78 & -0.786 & 0.702 & 0.101 & 0.158 & 0.017 & 0.016 & 0.004 & 0.008 \\ 0.012 & 0.012 & 0.012 & 0.012 & 0.017 & 0.015 & 0.019 & 0.016 & -0.942 & 0.78 & -1.143 & 0.67 & 0.088 & 0.141 & 0.015 & 0.015 & 0.003 & 0.008 \\ & & & & & & & & & & & & & & & & & \\ & & & & & & & & & & & & & & & & & & \\ 0.038 & 0.031 & 0.036 & 0.029 & 0.038 & 0.03 & 0.037 & 0.029 & -0.068 & 0.805 & -0.179 & 0.699 & 0.181 & 0.256 & 0.057 & 0.053 & 0.02 & 0.049 \\ 0.034 & 0.027 & 0.032 & 0.025 & 0.036 & 0.026 & 0.036 & 0.026 & -0.27 & 0.848 & -0.493 & 0.751 & 0.171 & 0.229 & 0.051 & 0.046 & 0.018 & 0.042\end{array}$




\section{Table 3 (on next page)}

Posterior probabilities of the speciation models

$n$ : number of individuals analyzed in each species; technique: rna sequencing ("rna-seq") vs. exome enrichment sequencing ("exome capture"); statistics: jsfs=4 (4 classes), jsfs=7 (7 classes), jsfs $=23$ (23 classes); $P P$ : posterior probability; $\mathrm{BF}_{1 / 2}:$ Bayes Factor defined as $\mathrm{PP}_{\text {best }}$ model $/ \mathrm{PP}_{2 \text { nd best model }} ; \mathrm{BF}_{1 / 3}$ : Bayes Factor defined as $\mathrm{PP}_{\text {best model }} / \mathrm{PP}_{\text {3rd best model }} 11$ models: $\mathrm{SI}, \mathrm{IM}$ hetero, IM homo, AM homo, AM hetero, PAM homo, PAM hetero, SC homo, SC hetero, PSC hetero, PSC homo. 
(A) 11 models

$\mathrm{n}=2$

$\mathrm{n}=4$

$\mathrm{n}=8$

$\mathrm{n}=2$

$\mathrm{n}=4$

$\mathrm{n}=8$

technique statistics

scenario

PP $\quad \mathrm{BF}_{1 / 2} \mathrm{BF}_{1 / 3} \quad$ scenario

PP $\quad \mathrm{BF}_{1 / 2} \mathrm{BF}_{1 / 3} \quad$ scenario

PP $\quad \mathrm{BF}_{1 / 2} \mathrm{BF}_{1 / 3}$ scenario

PP $\quad \mathrm{BF}_{1 / 2}$ scenario

(B) homo vs hetero for the best model exome $\quad$ jsf $=4$

exome

jsfs $=4$

$\begin{array}{llll}\text { PAM homo } & 0.360 & 1.45 & 1.87\end{array}$

PAM homo $0.340 \quad 1.33 \quad 1.70$

$\begin{array}{llll}\text { PAM homo } & 0.334 & 1.14 & 2.15\end{array}$

homo $\quad 0.557 \quad 1.26$

homo $0.695 \quad 2.27$

homo $\quad 0.538 \quad 1.16$

homo $\quad 0.519 \quad 1.08$

homo $0.509 \quad 1.04$

jsfs $=23$

$\begin{array}{lllllllll} & \text { PAM homo } & 0.309 & 1.26 & 1.34 & \text { PAM homo } & 0.250 & 1.17 & 1.21\end{array}$

PAM homo $0.337 \quad \begin{array}{lll}1.33 & 2.15\end{array}$

hetero $0.990 \quad 99$

hetero 0.982

homo $0.551 \quad 1.23$

hetero $1.000 \mathrm{NA}$ jsfs $=4 \quad$ PAM hetero $0.227 \quad 1.14 \quad 1.60 \quad$ PAM hetero $0.180 \quad 1.08 \quad 1.14$

rna-seq jsfs $=7$

jsfs $=23$ $\begin{array}{lllllll}\text { PAM hetero } 0.323 & 1.62 & 2.27 & \text { PAM hetero } 0.339 & 1.43 & 2.15\end{array}$

$\begin{array}{llllll}\text { PSC hetero } 0.414 & 1.07 & 2.60 & \text { PSC hetero } 0.346 & 1.37 & 1.47\end{array}$ hetero $0.556 \quad 1.25 \quad$ hetero $0.575 \quad 1.35$

hetero $0.733 \quad 2.75$ homo $0.520 \quad 1.08$

hetero $0.818 \quad 4.49$ hetero 0.997323 .33 